\title{
Nanotechnology: The Risks and Benefits for Medical Diagnosis and Treatment
}

\author{
Anderson DS, Sydor MJ, Fletcher P and Holian A* \\ Center for Environmental Health Sciences, Biomedical and Pharmaceutical Sciences, University of Montana, Missoula, MT, USA
}

*Corresponding author: Andrij Holian, Center for Environmental Health Sciences, Biomedical and Pharmaceutical Sciences, University of Montana, Missoula, MT, USA, E-mail: andrij.holian@umontana.edu

Received date: July 28, 2016; Accepted date: July 30, 2016; Published date: August 02, 2016

Copyright: (c) 2016 Anderson DS, et al. This is an open-access article distributed under the terms of the Creative Commons Attribution License, which permits unrestricted use, distribution, and reproduction in any medium, provided the original author and source are credited.

\section{Editorial}

Nanotechnology is being actively developed for many applications in the medical field, including drug delivery, biosensors and medical imaging. These nanomaterials are being advanced as novel and more targeted treatments for difficult to manage diseases such as cancers. Other materials are being developed as alternatives to conventional antibiotics in treating infections. The use of engineered nanomaterials (nanoparticles) offer the ability to transport therapeutics to specific sites of a disease, thus reducing the off target toxicity of many drugs. This is especially true in the use of chemotherapeutics where off target reactions cause serious side effects in cancer patients. Additionally, the field of medical imaging can be improved with the ability for the specific targeting of diseased tissues at resolutions not capable with current technologies.

As mentioned above, one of the primary uses of nanotechnology in the medical field has been the area of targeted drug delivery. It is crucial to deliver a drug to a desired target site in a controlled manner while not causing additional adverse health effects to the patient. To date the principle type of particle used in drug delivery systems is composed of lipids or polymers chosen for their biocompatibility. These systems have been used to deliver therapeutics more targeted or efficiently. For instance, nanospheres conjugated with disease specific antibodies or peptides can result in greatly increased local doses of treatments to sites of disease while avoiding high systemic levels of the therapeutic [1]. Biocompatible nanospheres have also been used to transport therapeutics with poor bioavailability. For instance, biologic treatments such as insulin and calcitonin, that cannot be delivered by conventional methods as an oral treatment, have successfully been packaged in hollow nanoparticles that protect it from degradation in the gastrointestinal tract allowing for systemic delivery of the drug and avoiding alternative methods of delivery such as subcutaneous injection [2]. However, there has also been a trend to use nanoparticles in medicine that have been produced from materials that are regarded as less biocompatible than those discussed above.

Nanoparticles have become a significant interest as a drug delivery system due to their small size and large surface area. The small sizes of nanoparticles increase efficacy for accurate intracellular uptake of the drug in the desired cellular targets and for accurate biodistribution $[3,4]$. The large surface area makes it easy to manipulate the particle into carrying high levels of drug or other compounds with ease [5]. Another important factor is the structural stability of nanoparticles to effectively deliver the drug over a long period of time without degradation occurring before it reaches the cellular target. In breast cancer treatment, Sabzichi, et al. used nanostructured lipid carriers to carry melatonin in human breast cancer cells which inhibited tumor proliferation and induced apoptosis. The study results indicated that the nano-carriers had effective biocompatibility and low cytotoxicity [5].

In contrast to the beneficial outcomes, using nanoparticles for drug delivery raises various safety concerns. The small size is beneficial, but it could have negative effects. This is a particularly important point as new and more durable materials are used in the production of these nanoparticles. Some nanoparticles can cause inflammation and fibrosis as a result of causing phagolysosomal membrane permeability, formation of reactive oxygen species and activation of the NLRP3 inflammasome [6]. The small size, in turn, indicates a large surface area which could be harmful by exposing more surface molecules to cellular components. Another concern is the preparation and stabilization processes of the nanoparticles for drug delivery since chemical reducing agents and radiation can exacerbate cytotoxicity. One antimicrobial testing study addressed this concern. Pradeepa, et al. used an environmentally friendly technique by means of bacterial exopolysaccharide to synthesize gold nanoparticles. They found that this new technique did not interfere with the antibiotic-coated gold nanoparticles ability to inhibit Gram negative and Gram positive bacteria activity [7]. Many things are still uncertain when using nanoparticles as a drug delivery system, which is why more research needs to be performed at relevant doses.

Nanoparticles have been proposed for improved systems in medical imaging for disease diagnosis. Much of the potential for nanomaterials as diagnostic agents comes from their ability to enhance contrast in spectroscopy. In particular, superparamagnetic iron oxide has been shown to enhance magnetic resonance imaging and as a result can aid in the detection of liver metastases [8]. Angiogenesis is a key hallmark of cancer and thus its detection would be of importance. Nanoparticles can be used for targeting sites of angiogenesis and enhancing diagnostic imaging. For example, cyanoacrylate microbubbles can be conjugated to ligands specific to biomarkers, such as vascular endothelial growth factor receptor and $\alpha v \beta 3$ integrin, which are more abundant with increased angiogenesis [9]. This conjugation allows for ultra sound detection of tumor phenotypes and quantification of these biomarkers, as well as, indicating responses to treatment.

Even though there are various applications for nanomaterials as diagnostic agents, some considerations need to be made with regard to their pharmacokinetic properties. Diagnostic agents must possess high target site specificity, low toxicity, and rapid clearance for the fraction unbound to the target. The size of the nanomaterial is also an important consideration. Agents larger than $5 \mathrm{~nm}$ will have much slower renal clearance than smaller agents. On the other hand, materials larger than $1 \mu \mathrm{m}$ will not undergo renal clearance and can, as a result, have high target specificity with a smaller unbound fraction needing to be cleared [10]. 
Citation: Anderson DS, Sydor MJ, Fletcher P, Holian A (2016) Nanotechnology: The Risks and Benefits for Medical Diagnosis and Treatment. J Nanomed Nanotechnol 7: e143. doi:10.4172/2157-7439.1000e143

Page 2 of 2

With the number and combination of materials used and the many different shapes and structures in which nanoparticles are being produced increasing each year there is a vital need to determine the properties of nanoparticles that may produce adverse reactions in patients receiving these materials as part of diagnosis or treatment. For instance, quantum dots have been shown to have properties that could improve medical imaging in the diagnosis of cancer [11]. However, quantum dots can contain cadmium, selenium, arsenic, and lead. While these particles have many advantages in diagnostics and imaging due to the unique properties that make them stand out from the biological background, these toxic metals are of concern [12]. Another example is the use of silver nanoparticles as an antibiotic to treat infection of multiple-drug resistant bacteria. Ionized silver from the silver nanoparticles has been found to be persistent in the organs of animals exposed and the long term effects of this persistent silver is poorly understood [13].

While nanotechnology, including the medical use of nanoparticles, hold great promise to improve the diagnosis and treatment of many diseases, we must not lose sight of the necessity to thoroughly test the nanomaterials so that they do not create unexpected adverse effects. This will require a balance between the safety of the materials used and the efficacy of the treatment. Different considerations of toxicology should be used when proposing that a given nanoparticle be used for a treatment of stage four cancer as compared to one to be used for a routine diagnostic medical imaging. Therefore, a situational approach should be used when assessing the benefits and drawbacks to using nanoparticles in medical diagnosis and treatment.

\section{References}

1. Sonaje K, Lin KJ, Wey SP, Lin CK, Yeh TH, et al. (2010) Biodistribution, pharmacodynamics and pharmacokinetics of insulin analogues in a rat model: Oral delivery using $\mathrm{pH}$-responsive nanoparticles vs. subcutaneous injection. Biomaterials 31: 6849-58.

2. Pridgen E, Alexis F, Farokhzad OC (2015) Polymeric Nanoparticle Drug Delivery Technologies for Oral Delivery Applications. Expert opinion on drug delivery 12: 1459-1473.
3. Bao H, Zhang Q, Xu H, Yan Z (2016) Effects of nanoparticle size on antitumor activity of 10-hydroxycamptothecin-conjugated gold nanoparticles: in vitro and in vivo studies. Int J Nanomedicine. 11: 929-40.

4. Semete B, Booysen L, Lemmer Y, Kalombo L, Katata L, et al. (2010) In vivo evaluation of the biodistribution and safety of PLGA nanoparticles as drug delivery systems. Nanomedicine 6: 662-71.

5. Sabzichi M, Samadi N, Mohammadian J, Hamishehkar H, Akbarzadeh M, et al. (2016) Sustained release of melatonin: A novel approach in elevating efficacy of tamoxifen in breast cancer treatment. Colloids Surf B Biointerfaces 145: 64-71.

6. Hamilton RF, Buford M, Xiang C, Wu N, Holian A (2012) NLRP3 inflammasome activation in murine alveolar macrophages and related lung pathology is associated with MWCNT nickel contamination. Inhalation Toxicology 24: 995-1008.

7. Pradeepa, Vidya SM, Mutalik S, Udaya Bhat K, Huilgol P, et al. (2016) Preparation of gold nanoparticles by novel bacterial exopolysaccharide for antibiotic delivery. Life Sci 15: 171-179.

8. Rappeport ED, Loft A, Berthelsen AK, von der Recke P, Larsen PN, et al. (2007) Contrast-enhanced FDG-PET/CT vs. SPIO-enhanced MRI vs. FDG-PET vs. CT in patients with liver metastases from colorectal cancer: a prospective study with intraoperative confirmation. Acta Radiol 48: 369-78.

9. Palmowski M, Huppert J, Ladewig G, Hauff P, Reinhardt M, et al. (2008) Molecular profiling of angiogenesis with targeted ultrasound imaging: early assessment of antiangiogenic therapy effects. Mol Cancer Ther 7: 101-9.

10. Baetke SC, Lammers T, Kiessling F (2015) Applications of nanoparticles for diagnosis and therapy of cancer. Br J Radiol 88: 20150207.

11. Santra S, Dutta D, Walter GA, Moudgil BM (2005) Fluorescent Nanoparticle Probes for Cancer Imaging. Technology in Cancer Research and Treatment 4: 593-602.

12. Pericleous P, Gazouli M, Lyberopoulou A, Rizos S, Nikiteas N, et al. (2012) Quantum dots hold promise for early cancer imaging and detection. International Journal of Cancer 131: 519-528.

13. Lee JH, Kim YS, Song KS, Ryu HR, Sung JH, et al. (2013) Biopersistence of silver nanoparticles in tissues from Sprague-Dawley rats. Particle and Fibre Toxicology 10: 36-36. 